This document is the accepted manuscript version of the following article: Campanini, M., Gradauskaite, E., Trassin, M., Yi, D., Yu, P., Ramesh, R., ... Rosse11, M. D. (2020). Imaging and quantification of charged domain walls in BiFe03. Nanoscale, 12(16), 9186-9193. https://doi.org/10.1039/D0NR01258K

\title{
Imaging and quantification of charged domain walls in $\mathrm{BiFeO}_{3}$.
}

\author{
Marco Campanini, ,a Elzbieta Gradauskaite, ${ }^{\mathrm{b}}$ Morgan Trassin, ${ }^{\mathrm{b}} \mathrm{Di} \mathrm{Yi},{ }^{\mathrm{c}} \mathrm{Pu}$ Yu, ${ }^{\mathrm{d}}$ Ramamoorthy Ramesh, ${ }^{\mathrm{e}}$ Rolf \\ Erni, $^{a}$ and Marta D. Rossell ${ }^{a}$
}

Charged domain walls in ferroelectrics hold great promise for the design of novel electronic devices due to their enhanced local conductivity. In fact, charged domain walls show unique properties including the possibility of being created, moved and erased by an applied voltage. Here, we demonstrate that the charged domain walls are constituted by a core region where most of the screening charge is localized and such charge accumulation is responsible for their enhanced conductivity. In particular, the link between the local structural distortions and charge screening phenomena in $109^{\circ}$ tail-to-tail domain walls of $\mathrm{BiFeO}_{3}$ is elucidated by a series of multiscale analysis performed by means of scanning probe techniques, including conductive atomic force microscopy (CAFM) and atomic resolution differential-phase contrast scanning transmission electron microscopy (DPC-STEM). The results prove that an accumulation of oxygen vacancies occurs at the tail-to-tail domain walls as the leading charge screening process. This work constitutes a new insight in understanding the behavior of such complex systems and lays down the fundaments for their implementation into novel nanoelectronic devices.

\footnotetext{
a. Electron Microscopy Center, Empa, Swiss Federal Laboratories for Materials Science and Technology, Ueberlandstr. 129,8600 Dübendorf, Switzerland

b. Laboratory for Multifunctional Ferroic Materials, ETHZ, 8093 Zürich, Switzerland

c. State Key Laboratory of New Ceramics and Fine Processing, School of Materials Science and Engineering, Tsinghua University, Beijing 100084, China.

d. State Key Laboratory of Low Dimensional Quantum Physics and Department of Physics, Tsinghua University, Beijing, 100084, China

e. Department of Materials Science and Engineering and Department of Physics, UC Berkeley, Berkeley, California 94720, USA.

Electronic Supplementary Information (ESI) available.

See DOI: $10.1039 / \times 0 \times x 00000 x$
}

\section{Introduction}

Domain walls (DWs) in ferroelectric materials constitute the two-dimensional (2D) boundary region between domains characterized by different values of the order parameter, i.e. the electrical polarization $(P)$. One of the most striking properties of DWs is their ability to carry a bound charge, due to the screening by the free carriers of the material. ${ }^{1}$ Although an intrinsic conductance due to bandgap lowering has been recently reported for formally uncharged head-to-tail $(\mathrm{H}-\mathrm{T})$ domain walls, ${ }^{2}$ the tail-to-tail $(\mathrm{T}-\mathrm{T})$ and headto-head $(\mathrm{H}-\mathrm{H})$ domain walls respectively hold a negative or positive nominal charge that can only be partially screened either by electrons, holes or mobile ions, provided by structural defects or doping. Therefore, the DWs that show non-zero bound charge are commonly named "charged domain walls". Following a recently introduced terminology, ${ }_{1}^{1}$ the T-T and $\mathrm{H}-\mathrm{H}$ DWs can be referred to as "strongly-charged" DWs in opposition to the "weakly-charged" H-T DWs. These charging phenomena can happen in the case that the adjacent domains are not conductive, e.g. in ferroelectric oxides. In such a case, the domain wall can be seen as a $2 \mathrm{D}$ conductive ${ }^{3-7}$ or superconductive ${ }^{8}$ pathway embedded in an insulating media. This special feature has raised an enormous interest in conductive DWs for their potential impact on future nanoelectronics. $^{9-14}$ In particular, as the domain walls in ferroelectric oxides are often movable under an external electric field, ${ }^{15-17}$ they represent a very promising element for novel rewritable circuitry.

Despite extensive research activities triggered by the unique behavior of charged DWs, their local structure and the nanoscale charge screening phenomena are still elusive. Indeed, charged domain walls and their properties have been investigated in various polycrystalline specimens and thin films, e.g. $\mathrm{BiFeO}_{3}{ }^{2,3,6,18} \mathrm{La}^{-}$ doped $\mathrm{BiFeO}_{3}{ }_{1}^{4} \mathrm{BaTiO}_{3},{ }^{15,19} \mathrm{LiNbO}_{3},{ }^{20} \mathrm{~Pb}\left(\mathrm{Zr}_{0.2} \mathrm{Ti}_{0.8}\right) \mathrm{O}_{3},{ }^{21,22} \mathrm{YMnO}_{3},{ }^{23-26} \mathrm{ErMnO}_{3} .{ }^{27-29} \mathrm{Ab}$ initio calculations suggest that charged domain walls cannot exist without charge screening promoted by the mobile charges, but the great modeling efforts of these complex structures still lack experimental validation. ${ }^{30}$ Moreover, the presence of charged defects, as e.g. oxygen vacancies, can promote - in addition to DW stabilization due to charge compensation - other important phenomena, like e.g. domain wall pinning. ${ }^{31}$ 
The deepening of our knowledge about the charge screening processes is of fundamental relevance to the achievement of a comprehensive understanding of the conduction properties of charged DWs. In ferroelectric oxides, however, the identification of the charge screening mechanisms is strongly puzzling because of the different possible structural defects. Moreover, the lack of measurement techniques that are directly sensitive to the localized charges with sub-nanometer resolution makes the identification and the quantitative estimation of the screening charges a challenging problem.

Here, we image and quantify at the atomic scale the charged defects distribution at the origin of the enhanced conductivity in charged domain walls. To do so, we studied conductive $109^{\circ} \mathrm{T}-\mathrm{T}$ domain walls in the archetype ferroelectric $\mathrm{BiFeO}_{3}$ by combining state-of-the-art scanning probe techniques - such as piezo-response force microscopy (PFM) and conductive atomic force microscopy (cAFM) - with the differential-phase contrast (DPC) scanning transmission electron microscopy (STEM) technique.

\section{Experimental}

\section{Thin Film Growth and Specimen Preparation}

The $\mathrm{BiFeO}_{3}(120 \mathrm{~nm}) / \mathrm{La}_{0.7} \mathrm{Sr}_{0.3} \mathrm{MnO}_{3}(12 \mathrm{uc}, \mathrm{t} \approx 5 \mathrm{~nm}) / \mathrm{SrRuO}_{3}(2.5 \mathrm{uc}, \mathrm{t} \approx 8 \AA) / \mathrm{STO}$ heterostructure was grown by pulsed laser deposition (PLD). Before the growth of the thin films, low-miscut $\mathrm{SrTiO}_{3}$ (STO) (001) substrates $\left(5 \times 5 \mathrm{~mm}^{2}\right)$ were wet-etched by buffered $\mathrm{HF}$ acid, followed by a thermal annealing process, forming the $\mathrm{TiO}_{2}$-terminated atomically flat surface. Precise control of thickness at the atomic scale was achieved by using in-situ reflection high-energy electron diffraction (RHEED). An ultrathin $\mathrm{SrRuO}_{3}$ (2.5 unit cells) was initially deposited on the STO substrate by counting two RHEED oscillations, to switch the termination from $\mathrm{TiO}_{2}$ to $\mathrm{SrO}$. $\mathrm{SrRuO}_{3}$ was deposited at $923 \mathrm{~K}$ in 100 mTorr of oxygen pressure. The repetition rate was $1 \mathrm{~Hz}$. Both the $\mathrm{La}_{0.7} \mathrm{Sr}_{0.3} \mathrm{MnO}_{3}(\mathrm{LSMO})$ and $\mathrm{BiFeO}_{3}$ (BFO) layers were grown at $963 \mathrm{~K}$ in $150 \mathrm{mTorr}$ of oxygen pressure. The laser fluence was $0.8 \mathrm{~J} \mathrm{~cm}^{-2}$. The repetition rate was $1 \mathrm{~Hz}$ for LSMO. The growth of LSMO was insitu monitored by counting 12 RHEED oscillations. BFO was deposited at a repetition rate of $3 \mathrm{~Hz}$ for 40 mins. After the growth, samples were cooled to room temperature under an oxygen pressure of 760 Torr at a rate of $5 \mathrm{~K} / \mathrm{min}$. Electron transparent cross-sectioned samples for transmission electron microscopy were prepared by means of an FEI Helios NanoLab 450S focused ion beam operated at accelerating voltages of 30 and $5 \mathrm{kV}$. Plasma cleaning $\left(25 \% \mathrm{O}_{2}, 75 \% \mathrm{Ar}\right)$ was performed for $120 \mathrm{~s}$ on the specimens before conducting the STEM experiments.

\section{Scanning Transmission Electron Microscopy}

The scanning transmission electron microscopy (STEM) experiments were carried out on an aberrationcorrected Titan Themis 80-300 operated at $300 \mathrm{kV}$, setting a probe semi-convergence angle of $18 \mathrm{mrad}$ and collecting semi-angles of 90-170 mrad for high angle annular dark-field (HAADF) and 10-40 mrad for the segmented (4-quadrant) annular dark field detector (DF4). The HAADF images were obtained through aligning and averaging image-series (20 frames, 2048x2048) by means of SmartAlign ${ }^{32}$ software, in order to increase the signal-to-noise ratio and to correct for the scan distortions. The specimen thickness was measured performing electron energy loss spectroscopy (EELS) experiments on a JEOL JEM-2200FS (200 $\mathrm{kV}$ ) equipped with an in-column Omega Filter. The thickness in the region of interest of the specimen was estimated to be $35 \pm 5 \mathrm{~nm}$, by means of the $\log$-ratio ${ }^{33}$ approach.

\section{Differential-Phase Contrast STEM}

The DPC technique is a STEM-based method able to detect the variations of the intensity distribution in the transmitted disk. Such intensity redistribution can be properly interpreted under a quantum mechanical description that relates the variation of the center of mass of the transmitted disk to the expectation value of the momentum. ${ }^{34}$ Moreover, the channeling effects also play a pivotal role in the beam propagation and consequently in the variation of the center of mass of the transmitted disk. ${ }^{35}$ Although as said a proper description of the signal generation requires a dynamical treatment of the beam propagations, such intensity redistribution can be intuitively comprehended if we picture the effect of the electric field in the specimen plane as a Lorentz force induced deflection of the electrons.DPC investigation was carried out using a probe-forming aperture semi-angle of $18 \mathrm{mrad}$ and a collection angular range for the segmented DF4 detector set to 10-40 mrad. The DPC measurements were performed by recording the images generated by the 4-quadrant DF4 detector and then computing the differential signals between opposite pairs of segments. The generated DPC signals are called DPC $(A-C)$ and DPC (B-D). The DPC signals were then differentiated to obtain a two-dimensional map of the projected charge density and integrated to retrieve the projected electrostatic potential. The signals so obtained are called differential- and integrated-DPC (dDCP and $i D P C)$, respectively. The DPC data processing and differentiation/integration were performed using custom-developed MATLAB scripts. 
The experimental data were de-noised using a custom-developed iterative non-linear algorithm based on the method proposed by Du et al. ${ }^{36}$ The precise fitting of the atomic columns in the STEM signals is strictly needed for the quantitative analysis of the structural distortions, ferroelectric polarization and the atomic column scattered intensities. In our analysis - similarly to the approaches reported in literature ${ }^{37,38}$ - we first evaluated the atomic column peak positions by means of a center of mass peak-finding algorithm and, afterward, we performed iterative refining of the fitted peaks solving a least-squares minimization problem (using the Levenberg-Marquardt algorithm). This refinement was carried out using custom-developed scripts that make use of 7-parameter two-dimensional Gaussians. The fitting allows a quantitative estimation of the atomic column peak intensities and their positions (with a precision of about $3 \mathrm{pm}$ ). After fitting the atomic column peaks, the strain analysis and polarization mapping were performed using a customdeveloped Peak-Pair ${ }^{39}$ algorithm. In all the figures, the error bars in the line-profiles are given as the standard deviation of the mean.

\section{Piezoresponse Force Microscopy and Conducive Atomic Force Microscopy}

Scanning probe microscopy measurements were carried out on an NTEGRA Prima scanning probe microscope from NT-MDT Spectrum Instruments. The PFM acquisition was performed using a $3 \mathrm{~V}$ peak-to-peak $\mathrm{AC}$ modulation at $70 \mathrm{kHz}$. CAFM images were recorded by measuring the current flow through the conductive tip with a DC bias voltage of up to $3.7 \mathrm{~V}$ applied. The measurements were performed using a lowcurrent Pt-coated tip. A region of the film was intentionally poled in order to perform a proper alignment of the specimen during the AFM and CAFM measurements. The PFM and CAFM signals were non-rigidly aligned using BigWarp plugin ${ }^{40}$ in ImageJ.

\section{Results and Discussion}

$\mathrm{BiFeO}_{3}$ thin films represent an ideal framework for the study of charged domain walls thanks to the possibility to precisely control the ferroelectric domain pattern by changing the growth parameters. ${ }^{41-43}$ Our study was conducted on the heterostructure constituted by $\mathrm{BiFeO}_{3}(120 \mathrm{~nm}) / \mathrm{La}_{0.7} \mathrm{Sr}_{0.3} \mathrm{MnO}_{3}(12 \mathrm{uc}, \mathrm{t} \approx 5$ $\mathrm{nm}) / \mathrm{SrRuO}_{3}(2.5 \mathrm{uc}, \mathrm{t} \approx 1.0 \mathrm{~nm}$ )/STO grown by pulsed laser deposition (PLD) in oxygen-rich conditions (see Experimental section for the details about the film deposition). In Fig. S1 (Supplementary Information) the XRD pattern of the structure is shown. The reflections in the $\theta-2 \theta$ scan prove that the film is a single-crystal and completely c-oriented and the substrate-induced epitaxial stress is relaxed by the formation of edge dislocations (see Fig. S2, Supplementary Information), as expected for the considered film thickness. ${ }^{44}$ The crystallographic structure with the c-axis oriented along the out-of-plane direction is compatible with two different domain configurations having the polarization along the $\langle 111\rangle_{\mathrm{p}}$ directions, respectively displaying $71^{\circ}$ and $109^{\circ}$ domain walls. The sketches of the two DWs for head-to-tail domains are given in Fig. 1a and $b$, respectively.

The polar domain pattern of the BFO film was studied by piezoresponse force microscopy (PFM) in both the lateral (LPFM) and vertical (VPFM) directions. The LPFM signal of a representative portion of the specimen is shown in Fig. $1 \mathrm{~d}$. The ferroelectric domain pattern displays the typical contrast of $71^{\circ} \mathrm{DWs}$ that appear on the surface as stripes oriented parallel to the main directions of the cubic substrate. This domain configuration leads to weakly-charged DWs, as the $71^{\circ}$ variation of the polarization vector occurs with respect to $\{101\}_{\mathrm{P}}$ or $\{011\}_{\mathrm{P}}$ planes, giving rise solely to $\mathrm{H}-\mathrm{T}$ configurations. Nevertheless, strongly-charged $\mathrm{H}-\mathrm{H}$ and T-T DWs are generated at the merging points of regions displaying domains separated by different sets of planes, as confirmed by the strong out-of-plane component of polarization visible in the VPFM signal shown in Fig. 1e.

Fig. If shows a magnified view of the region marked by the white box in Fig. $1 \mathrm{~d}$ and e, and can be directly related to its corresponding CAFM signal (Fig. 1g). In particular, the LPFM highlights two different types of charged domain walls, i.e. $109^{\circ} \mathrm{H}-\mathrm{H}$ and T-T DWs (marked by dotted and dashed lines, respectively). The DWs, which are $\{100\}_{p}$ type planes, separate domains whose polarization vectors are forming an angle of $109^{\circ}$. Fig. 1c shows the sketches of the charged $109^{\circ} \mathrm{T}-\mathrm{T}$ and $\mathrm{H}-\mathrm{H}$ DWs. In agreement with previous studies, ${ }^{3,4,45}$ the CAFM contrast confirms the strongly-conducting character of the charged domain wall. Remarkably, these charged domain walls display a significantly enhanced conductance with respect to the polar domains, thanks to their charged state.

By performing a focused ion beam (FIB) cut along one of the main directions of the STO substrate, the $109^{\circ}$ T-T DWs can be obtained in an electron transparent FIB lamella and investigated by scanning transmission electron microscopy (STEM). The analysis of the atomic positions and the atomic displacements in the HAADF signal allows the quantitative investigation of the structural and polar properties of the charged DW. More details about the fitting algorithms and data analysis are provided in the Materials and Methods section. 
A study of the local strain can be computed starting from the fitted position of the atomic columns by means of the Peak-Pairs (PP) algorithm (see Experimental section for details). The strain profiles, shown in Fig. $2 f$ for both the in-plane $\left(\varepsilon_{x x}\right)$ and out-of-plane $\left(\varepsilon_{y y}\right)$ directions, highlight a tensile in-plane strain $\left(\varepsilon_{x x} \approx\right.$ $2.3 \%$ in the core region of the DW. An analysis of the strain state of a larger portion of the $\mathrm{BiFeO}_{3}$ film is shown in Fig. S5 (Supplementary Information). The local variation in the HAADF intensity and the detected structural distortion suggest that the charge screening at the DW occurs with local accumulation of charged structural defects. Nevertheless, as no decrease in the Bi column intensities at the domain wall is observed, we can rule out an accumulation of $\mathrm{Bi}$ vacancies at the DW as the main charge screening mechanism.

The atomic-scale study of charge localization at the conductive DWs is of fundamental interest for gathering new insights on the relationship between their structural and functional properties. The ability of imaging and quantitatively estimate the charges at the DWs would enable us to tailor and engineer their properties by, e.g., strain or doping, paving the way to functional DW engineering. However, due to the complex domain wall structure that involves atomic displacements, strain and mixed nature of polarization switching, such understating still represents an open challenge. In particular, the quantitative estimation of the DW charges is mostly hindered by the lack of direct experimental techniques able to detect the localized charges with sub-nanometer resolution. In the following section, we provide a direct approach for the atomic-scale detection and quantification of localized charges.

The charged-state of the DW can be initially assessed by computing the density of bound charge that derives from the polar domain configuration. In particular, starting from the polarization maps obtained from the atomic displacements, we can calculate the internal electric field due to the remnant polarization of the material and then compute its divergence to extract the density of bound charges. Fig. 3a and $b$ show the two components of the electric field for the area displayed in Fig. 2a, while Fig. 3c shows a surface plot of the projected density of screening charges, $\rho_{\mathrm{B}}$. The plot highlights a distribution of positive charges mostly localized at the DW, with localized maxima separated a few nanometers from each other. The density of screening charges can be conveniently expressed by the number of elementary charges per unit cell $\left(\mathrm{ec} \cdot \mathrm{uc}^{-1}\right)$, in order to estimate the maximum amount of screening charges that each unit cell can accommodate. Taking the line profile over the region marked by the black dashed box, we can estimate the maximum charge for the localized peaks to be $0.25 \mathrm{ec} \cdot \mathrm{uc}^{-1}$. Assuming a fully-ionized state of oxygen vacancies - as expected due to the oxygen-rich grown conditions ${ }^{48}$ - we are able to estimate a maximum concentration of 1 oxygen vacancy $\left(\mathrm{O}_{\mathrm{v}}\right)$ every 4 unit cells on the localized peaks of $\rho_{B}$.

In order to accomplish a direct measurement of the charges within the DW, we performed differential-DPC (dDPC) imaging, which essentially maps the charge distribution in the sample. The DPC technique in either its differential or integrated forms (i.e. $\mathrm{dDPC}$ and $\mathrm{iDPC})^{49}$ - thanks to its enhanced sensitivity to low $\mathrm{Z}$ species - has been recently been proven to be effective for imaging of light elements, such as $\mathrm{N}^{50} \mathrm{Li}^{51} \mathrm{O},{ }^{52}$ and $\mathrm{H}_{1}{ }^{53}$ whose detection is challenging by conventional STEM imaging methods. Here, we employ the dDPC technique to detect the light $O$ columns in the BFO layer and to map the $O$ vacancy distribution.

Fig. 4 a illustrates the basic principles at the origin of differential-phase contrast in STEM measurements. ${ }^{54-}$ 56 The sketch shows that an electric field in the specimen plane induces intensity redistribution in the transmitted disk that can be detected by a segmented annular dark-field (ADF) detector (see Experimental section for more details about the DPC technique). The intensity variation on opposite pairs of segments allows for mapping such electric fields and, in turn, by differentiation and integration of the DPC signal we can calculate the projected density of charges and the projected potential, respectively.

The entire set of DPC signals obtained on an area across the DW is given in Fig. S6 of Supplementary Information (the details about data acquisition and processing are provided in the Experimental section). As visible in the dDPC signal, the increased sensitivity for low-Z elements allows the imaging of $O$ columns. By fitting the intensity of the $O$ columns, we can therefore visualize the oxygen vacancies distribution as the columns containing vacancies will appear with a lower intensity with respect to the fully occupied ones. A map of the $O$ column intensity is displayed in Fig. $4 d$, where the core of the charged DW is immediately evident as a region with a darker contrast due to the larger density of $O$ vacancies. Interestingly, some dark spots are visible along the DW. They recall the local maxima observed in the previously described map of the projected bound charge (Fig. 3c). As an example, one of these dark spots is marked with a white box in the intensity map of the O columns; a magnified view of the corresponding portion of the high-resolution dDPC image is also provided in Fig. 4e. From the relative intensities of the $O$ columns, it is evident that the dark spot corresponds to a local accumulation of $O$ vacancies along the $O$ column at the center of the image (marked by a white circle). By taking a line profile of dDPC across a $\mathrm{BiO}_{2}$ layer that contains one of these columns with highly vacant $O$ sites (the region used for the line profile is marked by a yellow box in Fig. S6, Supplementary Information), we can observe that the dDPC signal is significantly perturbed by the accumulation of $\mathrm{O}_{v}$ as shown in Fig. 4f. On the contrary, no variations in the fitted intensities for the A- and $B$-sites columns are observed (Fig. $4 b$ and $c$ ). These results prove that high-resolution dDPC STEM imaging 
is very effective in imaging light elements, like oxygen, and that it allows for detecting variations in their distribution along single atomic columns.

A similar analysis, but performed on the iDPC signal is shown in Fig. S7 of Supplementary Information. Despite the highly vacant O column can be similarly localized, the amplification of lower spatial frequencies (e.g. specimen surface contamination) hinders the proper identification of the $\mathrm{O}$ vacancies distribution at the charged DW.

\section{Conclusions}

In conclusion, the presented analysis of the strongly-charged T-T domain wall elucidates the nature of this structure, providing new insights about its structure and charge screening phenomena. The charge screening phenomena were studied by the DPC STEM technique that - thanks to its strong sensitivity to light atoms - is a very efficient tool to map the local distribution of oxygen vacancies. In particular, we show that combining atomic displacement mapping and DPC-STEM imaging we can map and quantitatively estimate the charge that is responsible for the high conductance of the $109^{\circ} \mathrm{T}-\mathrm{T} \mathrm{DW}$. Moreover, the proposed approach can be applied to study the charged state of buried ferroelectric domain walls, ${ }^{31,57}$ whose conductive properties are not accessible by surface scanning probe microscopies like CAFM.

Our results prove that the charged DW is composed of a localized core where most of the screening charge is localized. In the investigated case, i.e. $109^{\circ} \mathrm{T}-\mathrm{T}$ charged domain wall in $\mathrm{BiFeO}_{3}$, the screening is achieved by a local accumulation of oxygen vacancies in a region whose width is 6-8 unit cells. The accommodation of a high concentration of oxygen vacancies - corresponding to an average projected charge density of about 0.25 elementary charge per unit cell - in the DW core is allowed by a structural deformation (i.e. tensile strain) that in turn is responsible for a remarkable drop (about 50\%) of polarization at the DW core. The strong relationship between the structural strain and the charged defect accumulation suggests new possible routes to tailor the conductivity of charged domain walls. These findings represent a step forward in the understanding of the charge screening phenomena in functional oxides thin films and pave the way for the future development of novel ferroelectric devices that fully exploit the transport properties of charged domain walls.

\section{Conflicts of interest}

There are no conflicts to declare.

\section{Acknowledgements}

This research was supported by SNSF project no. 200021_175926. 
(a)
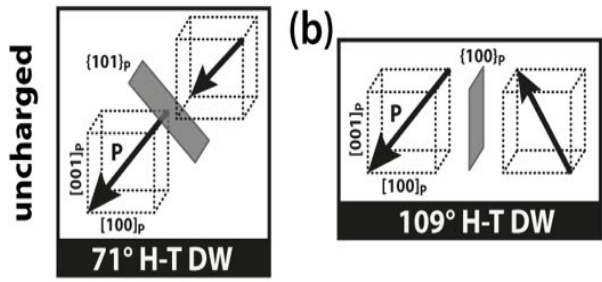

(c)

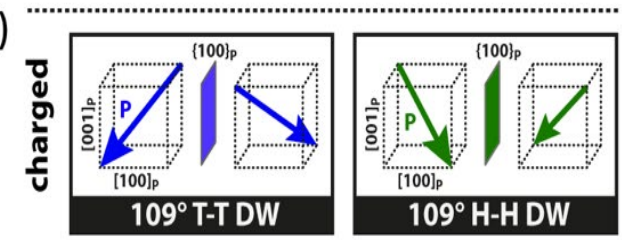

(d)
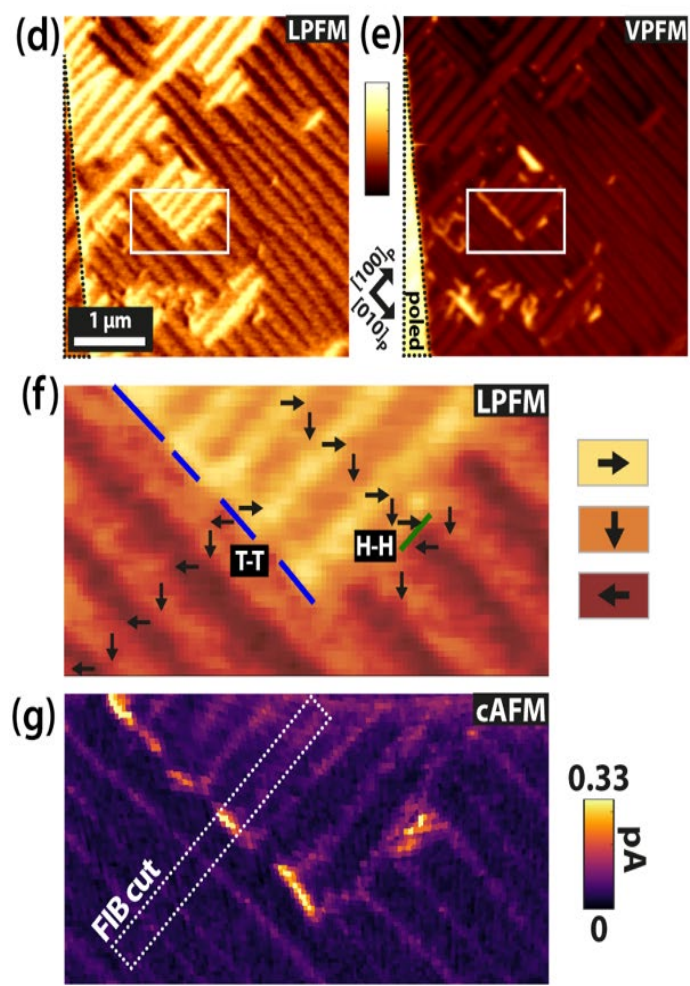

Fig. 1 (a) Sketches of the uncharged $71^{\circ}$ and (b) $109^{\circ} \mathrm{H}-\mathrm{T}$ DW configurations. The crystallographic directions and DWs planes are given in the pseudocubic reference. (c) Sketches of the $109^{\circ}$ $\mathrm{T}-\mathrm{T}$ and $\mathrm{H}-\mathrm{H}$ charged DWs. (d) LPFM image showing the distribution of polar domains in the film. The film shows a typical pattern of $71^{\circ}$ domains with four possible crystallographic variants. (e) VPFM signal of the same area. An out-of-plane signal is observed at the merging points between differently oriented domain stripes. The poled area (used as a reference for sample alignment) is marked in (d) and (e) by the black dashed line. (f) Magnified view of the area marked in the white box in (d) and (e), and (g) the corresponding CAFM signal. The arrows give the in-plane component of polarization. The configuration of the polar domains gives rise to regions where $109^{\circ} \mathrm{T}-\mathrm{T}$ and $\mathrm{H}-\mathrm{H}$ domain walls are observed. These DWs show a high conductance state compared to the low conductivity of H-T DWs. The white dashed box shows the area of a FIB cut that includes one charged T-T DW. 
(a)

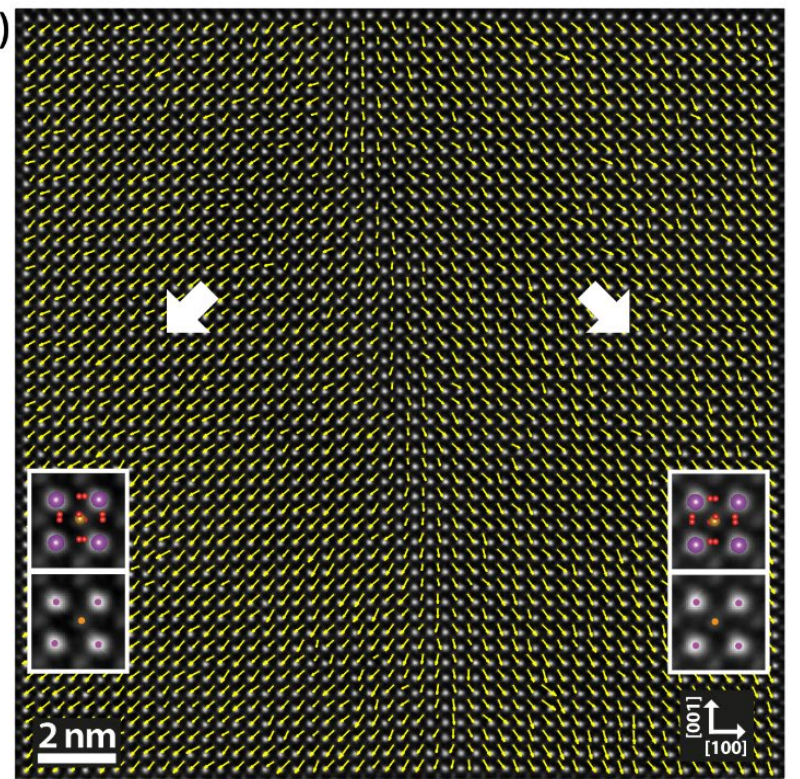

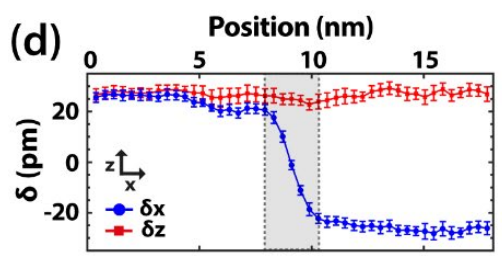

(e)

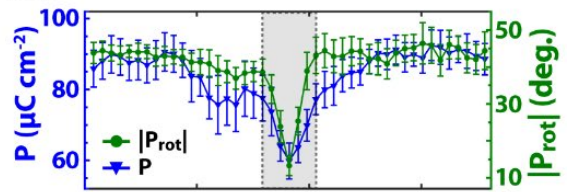

(f)

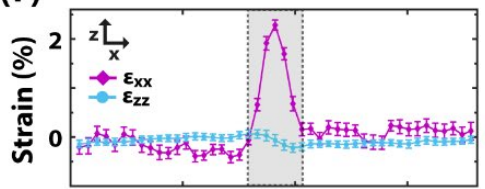

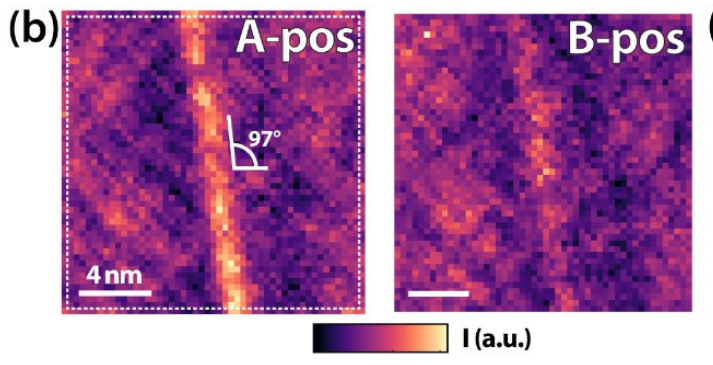
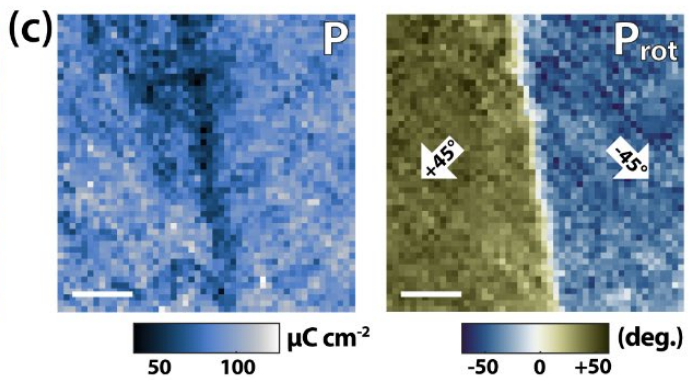

Fig. 2 (a) High-resolution HAADF-STEM image of the T-T charged domain wall in the $\mathrm{BiFeO}_{3}$ thin film. The magnified portions given in the inset show the pseudocubic structure at both sides of the DW. (b) Intensity maps of the A-and B-site atomic columns of the pseudocubic structure as obtained from the HAADF signal. The map of the B-cation intensity is obtained from the area marked by the white box in the A-cation intensity map. (c) Maps of the polarization amplitude (P) and polarization rotation ( $P_{\text {rot }}$ ). The rotation angle is given with respect to the vertical direction. ( $d-f$ ) Quantitative analysis of the structural distortions and polar instability across the domain wall region: (d) profiles of the atomic displacements along the [100]p and [001]p directions, (e) profiles of polarization amplitude and its net rotation angle, ( $f$ ) profiles of in-plane and out-of-plane strain components. The region corresponding to the domain wall core is marked by the grey box in $(d-f)$. 
(a)

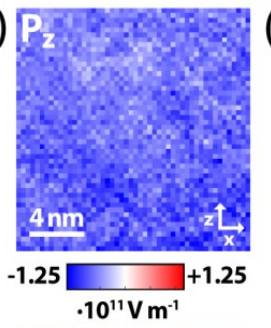

(b)

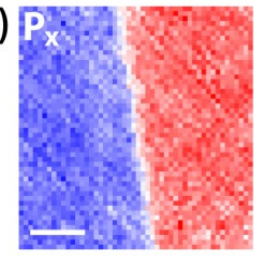

(c)
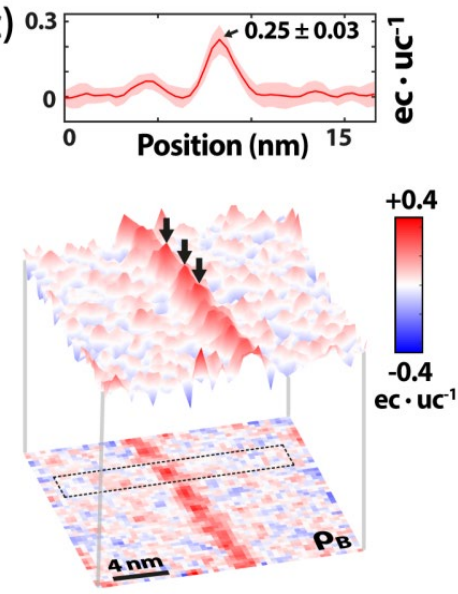

Fig. 3 (a) Map of the remnant polarization components $\left(P_{x_{1}} P_{z}\right)$ for the same portion of the specimen shown in Fig. 1a. The $x$ and $z$ directions correspond to the horizontal and vertical directions, respectively. The charged DW is noticeable in the $P_{x}$ map as the in-plane component of the polarization vanishes at the core region. (c) Surface plot of the screening charges density $\left(\rho_{B}\right.$, given in terms of elementary charges per unit cell) that highlights an accumulation of positive charges at the DW core. The profile taken in the region marked by the black dashed box allows estimating the maximum charge density that can accumulate at the DW core.

(a)
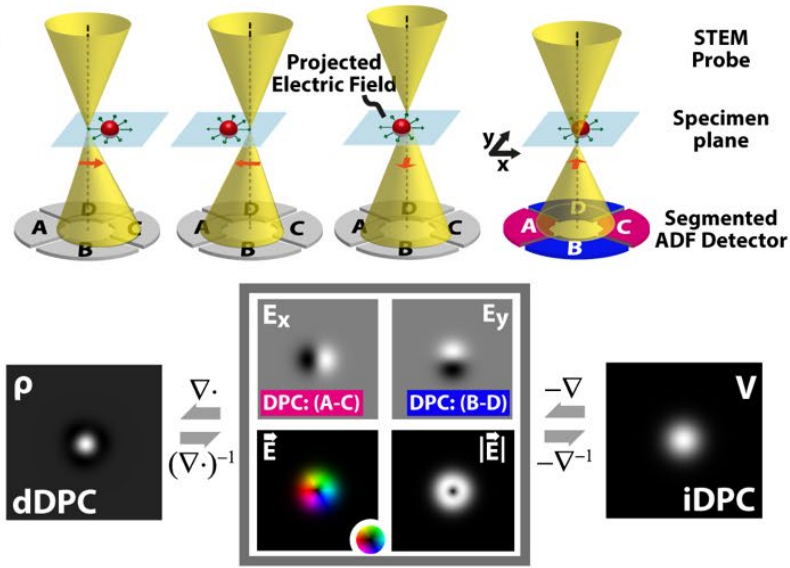

(b)
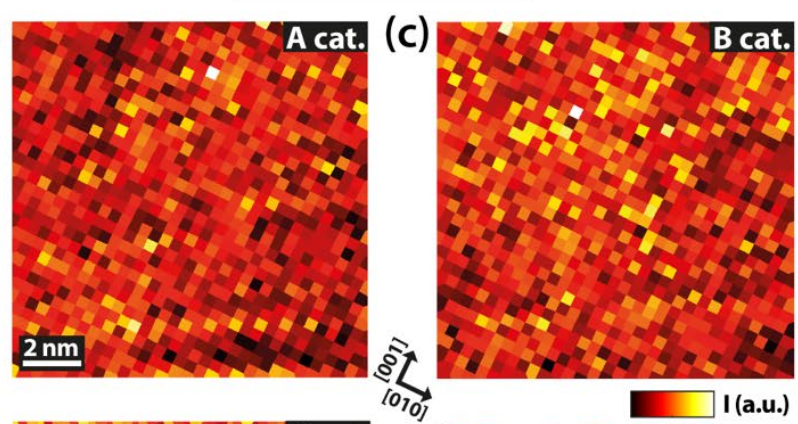

(d)
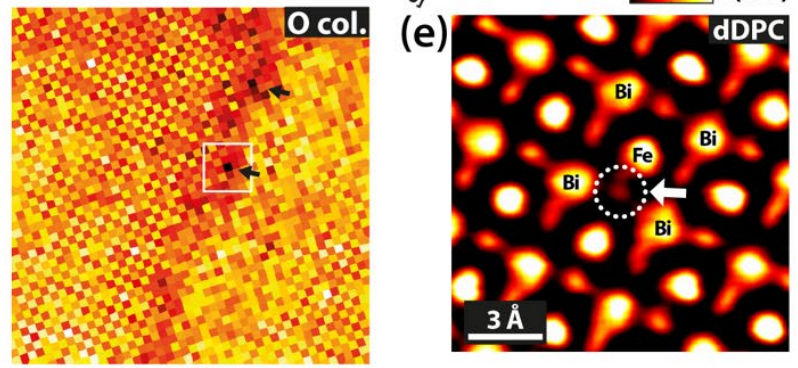

(e)

(f)

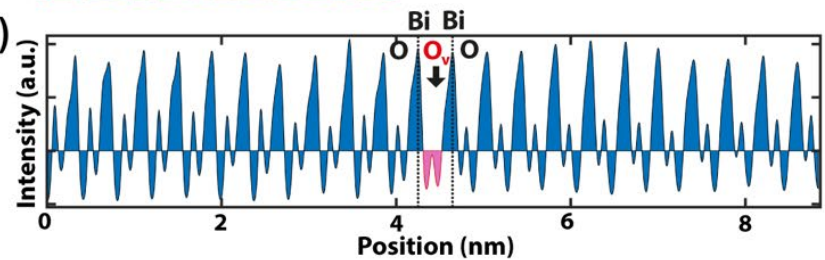

Fig. 4 (a) Simplified scheme of the basic principles of the DPC technique. The electric field in the specimen plane induces a deflection of the electron probes that is detected by means of a segmented annular detector, as the differential signals between opposite pairs of segments. The DPC signals can be differentiated (dDPC) or integrated (iDPC) to obtain the projected charge density and projected potentials, respectively. As an example, the panels show the signals generated by a single atom in vacuum. (b, c) Intensity maps of the A- and B-site atomic columns obtained from a high resolution $\mathrm{dDPC}$ micrograph, across a region containing the $109^{\circ} \mathrm{T}-\mathrm{T}$ DW. (d) Corresponding intensity map of the $O$ columns showing a reduced content of $O$ at the DW. The darker contrast at the DW can be attributed to a local accumulation of $O$ vacancies. The scale-bar for (c) and (d) is the same shown in (b). (e) Magnified view of the high-resolution dDPC signal that corresponds to the area delimited by the white box 


\section{References}

P. S. Bednyakov, B. I. Sturman, T. Sluka, A. K. Tagantsev and P. V. Yudin, npj Comput. Mater., 2018, 4, 65. Y. Zhang, H. Lu, X. Yan, X. Cheng, L. Xie, T. Aoki, L. Li, C. Heikes, S. P. Lau, D. G. Schlom, L. Chen, A. Gruverman and X. Pan, Adv. Mater., 2019, 31, 1902099.

J. Seidel, L. W. Martin, Q. He, Q. Zhan, Y. H. Chu, A. Rother, M. E. Hawkridge, P. Maksymovych, P. Yu, M. Gajek, N. Balke, S. V. Kalinin, S. Gemming, F. Wang, G. Catalan, J. F. Scott, N. A. Spaldin, J. Orenstein and R. Ramesh, Nat. Mater., 2009, 8, 229-234.

J. Seidel, P. Maksymovych, Y. Batra, A. Katan, S. Y. Yang, Q. He, A. P. Baddorf, S. V. Kalinin, C. H. Yang, J. C. Yang, Y. H. Chu, E. K. H. Salje, H. Wormeester, M. Salmeron and R. Ramesh, Phys. Rev. Lett., 2010, 105, 197603.

P. Maksymovych, J. Seidel, Y. H. Chu, P. Wu, A. P. Baddorf, L.-Q. Chen, S. V. Kalinin and R. Ramesh, Nano Lett., 2011, 11, 1906-1912.

T. Rojac, A. Bencan, G. Drazic, N. Sakamoto, H. Ursic, B. Jancar, G. Tavcar, M. Makarovic, J. Walker, B. Malic and D. Damjanovic, Nat. Mater., 2017, 16, 322-327.

J. A. Mundy, J. Schaab, Y. Kumagai, A. Cano, M. Stengel, I. P. Krug, D. M. Gottlob, H. Doğanay, M. E. Holtz, R. Held, Z. Yan, E. Bourret, C. M. Schneider, D. G. Schlom, D. A. Muller, R. Ramesh, N. A. Spaldin and D. Meier, Nat. Mater., 2017, 16, 622-627.

A. Aird and E. K. H. Salje, J. Phys. Condens. Matter, , DOI:10.1088/0953-8984/10/22/003.

J. Seidel, J. Phys. Chem. Lett., 2012, 3, 2905-2909.

E. K. H. Salje, ChemPhysChem, 2010, 11, 940-950.

J. Jiang, Z. L. Bai, Z. H. Chen, L. He, D. W. Zhang, Q. H. Zhang, J. A. Shi, M. H. Park, J. F. Scott, C. S. Hwang and A. Q. Jiang, Nat. Mater., 2017, 17, 49.

L. Li, J. Britson, J. R. Jokisaari, Y. Zhang, C. Adamo, A. Melville, D. G. Schlom, L.-Q. Chen and X. Pan, Adv. Mater., 2016, 28, 6574-6580.

P. Sharma, Q. Zhang, D. Sando, C. H. Lei, Y. Liu, J. Li, V. Nagarajan and J. Seidel, Sci. AdV., 2017, 3, e1700512.

G. Catalan, J. Seidel, R. Ramesh and J. F. Scott, Rev. Mod. Phys., 2012, 84, 119-156.

T. Rojac, H. Ursic, A. Bencan, B. Malic and D. Damjanovic, Adv. Funct. Mater., 2015, 25, 2099-2108.

J. C. Agar, A. R. Damodaran, M. B. Okatan, J. Kacher, C. Gammer, R. K. Vasudevan, S. Pandya, L. R. Dedon, R. V. K. Mangalam, G. A. Velarde, S. Jesse, N. Balke, A. M. Minor, S. V. Kalinin and L. W. Martin, Nat. Mater., 2016, 15, 549556.

R. J. Zednik, A. Varatharajan, M. Oliver, N. Valanoor and P. C. McIntyre, Adv. Funct. Mater., 2011, 21, 3104-3110.

S. Yang, R.-C. Peng, Q. He, Y.-L. Huang, Y. Huang, J.-C. Yang, T. Chen, J. Guo, L.-Q. Chen, Y.-H. Chu, C.-W. Nan and P. Yu, Ann. Phys., 2018, 530, 1800130.

T. Sluka, A. K. Tagantsev, P. Bednyakov and N. Setter, Nat. Commun., 2013, 4, 1808.

V. Y. Shur, E. L. Rumyantsev, E. V. Nikolaeva and E. I. Shishkin, Appl. Phys. Lett., 2000, 77, 3636-3638.

J. Guyonnet, I. Gaponenko, S. Gariglio and P. Paruch, Adv. Mater., 2011, 23, 5377-5382.

G. De Luca, M. D. Rossell, J. Schaab, N. Viart, M. Fiebig and M. Trassin, Adv. Mater., 2017, 29, 1605145.

T. Matsumoto, R. Ishikawa, T. Tohei, H. Kimura, Q. Yao, H. Zhao, X. Wang, D. Chen, Z. Cheng, N. Shibata and Y. Ikuhara, Nano Lett., 2013, 13, 4594-4601.

E. Ruff, S. Krohns, M. Lilienblum, D. Meier, M. Fiebig, P. Lunkenheimer and A. Loidl, Phys. Rev. Lett., 2017, 118, 036803.

D. R. Småbråten, Q. N. Meier, S. H. Skjærvø, K. Inzani, D. Meier and S. M. Selbach, Phys. Rev. Mater., 2018, 2, 114405.

Y. Du, X. L. Wang, D. P. Chen, S. X. Dou, Z. X. Cheng, M. Higgins, G. Wallace and J. Y. Wang, Appl. Phys. Lett., 2011, 99, 252107.

D. Meier, J. Seidel, A. Cano, K. Delaney, Y. Kumagai, M. Mostovoy, N. A. Spaldin, R. Ramesh and M. Fiebig, Nat. Mater., 2012, 11, 284-288.

P. Schoenherr, K. Shapovalov, J. Schaab, Z. Yan, E. D. Bourret, M. Hentschel, M. Stengel, M. Fiebig, A. Cano and D. Meier, Nano Lett., 2019, 19, 1659-1664.

P. W. Turner, J. P. V. McConville, S. J. McCartan, M. H. Campbell, J. Schaab, R. G. P. McQuaid, A. Kumar and J. M. Gregg, Nano Lett., 2018, 18, 6381-6386.

B. Sturman, E. Podivilov, M. Stepanov, A. Tagantsev and N. Setter, Phys. Rev. B, 2015, 92, 214112. W. R. Geng, X. H. Tian, Y. X. Jiang, Y. L. Zhu, Y. L. Tang, Y. J. Wang, M. J. Zou, Y. P. Feng, B. Wu, W. T. Hu and X. L. Ma, Acta Mater., 2020, 186, 68-76.

L. Jones, H. Yang, T. J. Pennycook, M. S. J. Marshall, S. Van Aert, N. D. Browning, M. R. Castell and P. D. Nellist, Adv. Struct. Chem. Imaging, 2015, 1, 8 .

T. Malis, S. C. Cheng and R. F. Egerton, J. Electron Microsc. Tech., 1988, 8, 193-200.

K. Müller, F. F. Krause, A. Béché, M. Schowalter, V. Galioit, S. Löffler, J. Verbeeck, J. Zweck, P. Schattschneider and A. Rosenauer, Nat. Commun., 2014, 5, 5653. 
R. Close, Z. Chen, N. Shibata and S. D. Findlay, Ultramicroscopy, 2015, 159, 124-137. H. Du, Ultramicroscopy, 2015, 151, 62-67. A. B. Yankovich, B. Berkels, W. Dahmen, P. Binev, S. I. Sanchez, S. A. Bradley, A. Li, I. Szlufarska and P. M. Voyles, Nat. Commun., 2014, 5, 4155.

A. De Backer, K. H. W. van den Bos, W. Van den Broek, J. Sijbers and S. Van Aert, Ultramicroscopy, 2016, 171, 104116.

P. L. Galindo, S. Kret, A. M. Sanchez, J. Y. Laval, A. Yáñez, J. Pizarro, E. Guerrero, T. Ben and S. I. Molina, Ultramicroscopy, 2007, 107, 1186-1193.

J. A. Bogovic, P. Hanslovsky, A. Wong and S. Saalfeld, in Proceedings - International Symposium on Biomedical Imaging, IEEE Computer Society, 2016, vol. 2016-June, pp. 1123-1126.

W. Y. Wang, Y. L. Zhu, Y. L. Tang, Y. B. Xu, Y. Liu, S. Li, S. R. Zhang, Y. J. Wang and X. L. Ma, Appl. Phys. Lett., , DOI:10.1063/1.4967878.

Y. Yun, N. Ramakrishnegowda, D. S. Park and A. Bhatnagar, Appl. Phys. Lett., , DOI:10.1063/1.5037512.

P. Yu, W. Luo, D. Yi, J. X. Zhang, M. D. Rossell, C. H. Yang, L. You, G. Singh-Bhalla, S. Y. Yang, Q. He, Q. M. Ramasse, R. Erni, L. W. Martin, Y. H. Chu, S. T. Pantelides, S. J. Pennycook and R. Ramesh, Proc. Natl. Acad. Sci. U. S. A., 2012, 109, 9710-9715.

P. Agrawal, M. Campanini, A. Rappe, S. Liu, V. Grillo, C. Hébert, R. Erni, D. Passerone and M. D. Rossell, Phys. Rev. Mater., 2019, 3, 034410.

W. Wu, Y. Horibe, N. Lee, S. W. Cheong and J. R. Guest, Phys. Rev. Lett., 2012, 108, 077203.

C.-L. Jia, S.-B. Mi, K. Urban, I. Vrejoiu, M. Alexe and D. Hesse, Nat. Mater., 2008, 7, 57-61.

A. Lubk, S. Gemming and N. A. Spaldin, Phys. Rev. B, 2009, 80, 104110.

G. Geneste, C. Paillard and B. Dkhil, Phys. Rev. B, , DOI:10.1103/PhysRevB.99.024104.

I. Lazić, E. G. T. Bosch and S. Lazar, Ultramicroscopy, 2016, 160, 265-280.

E. Yücelen, I. Lazić and E. G. T. Bosch, Sci. Rep., 2018, 8, 2676.

A. Carlsson, I. Alexandrou, E. Yücelen, E. G. T. Bosch and I. Lazić, Microsc. Microanal., 2018, 24, 122-123.

S. Findlay, N. Shibata, Y. Ikuhara, L. Clark, H. Brown, T. Petersen, D. Paganin and M. Morgan, Microsc. Microanal., 2019, 25, 1732-1733.

S. de Graaf, J. Momand, C. Mitterbauer, S. Lazar and B. J. Kooi, Sci. Adv., 2020, 6, eaay4312.

N. Shibata, S. D. Findlay, T. Matsumoto, Y. Kohno, T. Seki, G. Sánchez-Santolino and Y. Ikuhara, Acc. Chem. Res., 2017, 50, 1502-1512.

N. Shibata, T. Seki, G. Sánchez-Santolino, S. D. Findlay, Y. Kohno, T. Matsumoto, R. Ishikawa and Y. Ikuhara, Nat. Commun., 2017, 8, 15631.

T. Matsumoto, Y.-G. So, Y. Kohno, H. Sawada, Y. Ikuhara and N. Shibata, Sci. Adv., , DOI:10.1126/sciadv.1501280. M. Campanini, R. Erni, C. H. Yang, R. Ramesh and M. D. Rossell, Nano Lett., 2018, 18, 717-724. 